\title{
Rapid response air medical evacuation by civilian HEMS crew of critical patients during COVID-19 outbreak - First Fixed Points
}

\author{
Mauro Del Romano, ${ }^{1,2}$ Lorenzo Ciapessoni, ${ }^{1,2}$ Francesca Di Mola,, ${ }^{1,2}$ Gabriele Romanò,,2 \\ Massimo Gavezzotti, ${ }^{1,2}$ Marcello Rigamonti, ${ }^{1,2}$ Monica Carnelli, ${ }^{1,2}$ Roberto Cappelletti, ${ }^{1,2}$ \\ Elena Sala Peup, ${ }^{1,2}$ Edgardo Orlandi, ${ }^{1,2}$ Guido Francesco Villa,, ${ }^{1,2}$ Giordana Manuel, ${ }^{3}$ \\ Walter Ponzoni ${ }^{3}$ \\ ${ }^{1}$ Agenzia Regionale Emergenza Urgenza (AREU), Servizio regionale di elisoccorso, base di Villa Guardia; \\ ${ }^{2}$ AAT 118 Como; ${ }^{3}$ Helicopter pilots, Babcock Mission Critical Service Italia S.p.A., Italy
}

\begin{abstract}
The Covid-19 emergency requires a shared plan for the long-
range helicopter transfer of patients affected by the virus in order
to reduce the overload of intensive care units. To date, there is lim-
ited peer reviewed literature on aeromedical transport of patients
with highly hazardous communicable diseases, most of it is mili-
tary, and none deals specifically with patients affected by Covid-
19. To meet this need, we propose reference criteria regarding pre-
flight, in-flight and post-flight patient management and helicopter
sanitization.
Abstract
The Covid-19 emergency requires a shared plan for the long-
range helicopter transfer of patients affected by the virus in order
to reduce the overload of intensive care units. To date, there is lim-
ited peer reviewed literature on aeromedical transport of patients
with highly hazardous communicable diseases, most of it is mili-
tary, and none deals specifically with patients affected by Covid-
19. To meet this need, we propose reference criteria regarding pre-
flight, in-flight and post-flight patient management and helicopter
sanitization.

Abstract
The Covid-19 emergency requires a shared plan for the long-
range helicopter transfer of patients affected by the virus in order
to reduce the overload of intensive care units. To date, there is lim-
ited peer reviewed literature on aeromedical transport of patients
with highly hazardous communicable diseases, most of it is mili-
tary, and none deals specifically with patients affected by Covid-
19. To meet this need, we propose reference criteria regarding pre-
flight, in-flight and post-flight patient management and helicopter
sanitization.

Abstract
The Covid-19 emergency requires a shared plan for the long-
range helicopter transfer of patients affected by the virus in order
to reduce the overload of intensive care units. To date, there is lim-
ited peer reviewed literature on aeromedical transport of patients
with highly hazardous communicable diseases, most of it is mili-
tary, and none deals specifically with patients affected by Covid-
19. To meet this need, we propose reference criteria regarding pre-
flight, in-flight and post-flight patient management and helicopter
sanitization.

Abstract
The Covid-19 emergency requires a shared plan for the long-
range helicopter transfer of patients affected by the virus in order
to reduce the overload of intensive care units. To date, there is lim-
ited peer reviewed literature on aeromedical transport of patients
with highly hazardous communicable diseases, most of it is mili-
tary, and none deals specifically with patients affected by Covid-
19. To meet this need, we propose reference criteria regarding pre-
flight, in-flight and post-flight patient management and helicopter
sanitization.

Abstract
The Covid-19 emergency requires a shared plan for the long-
range helicopter transfer of patients affected by the virus in order
to reduce the overload of intensive care units. To date, there is lim-
ited peer reviewed literature on aeromedical transport of patients
with highly hazardous communicable diseases, most of it is mili-
tary, and none deals specifically with patients affected by Covid-
19. To meet this need, we propose reference criteria regarding pre-
flight, in-flight and post-flight patient management and helicopter
sanitization.

Abstract
The Covid-19 emergency requires a shared plan for the long-
range helicopter transfer of patients affected by the virus in order
to reduce the overload of intensive care units. To date, there is lim-
ited peer reviewed literature on aeromedical transport of patients
with highly hazardous communicable diseases, most of it is mili-
tary, and none deals specifically with patients affected by Covid-
19. To meet this need, we propose reference criteria regarding pre-
flight, in-flight and post-flight patient management and helicopter
sanitization.

Abstract
The Covid-19 emergency requires a shared plan for the long-
range helicopter transfer of patients affected by the virus in order
to reduce the overload of intensive care units. To date, there is lim-
ited peer reviewed literature on aeromedical transport of patients
with highly hazardous communicable diseases, most of it is mili-
tary, and none deals specifically with patients affected by Covid-
19. To meet this need, we propose reference criteria regarding pre-
flight, in-flight and post-flight patient management and helicopter
sanitization.

Abstract
The Covid-19 emergency requires a shared plan for the long-
range helicopter transfer of patients affected by the virus in order
to reduce the overload of intensive care units. To date, there is lim-
ited peer reviewed literature on aeromedical transport of patients
with highly hazardous communicable diseases, most of it is mili-
tary, and none deals specifically with patients affected by Covid-
19. To meet this need, we propose reference criteria regarding pre-
flight, in-flight and post-flight patient management and helicopter
sanitization.

Abstract
The Covid-19 emergency requires a shared plan for the long-
range helicopter transfer of patients affected by the virus in order
to reduce the overload of intensive care units. To date, there is lim-
ited peer reviewed literature on aeromedical transport of patients
with highly hazardous communicable diseases, most of it is mili-
tary, and none deals specifically with patients affected by Covid-
19. To meet this need, we propose reference criteria regarding pre-
flight, in-flight and post-flight patient management and helicopter
sanitization.
\end{abstract}

\section{Introduction}

In symptomatic patients, infection by SARS-CoV-2 causes fever and respiratory symptoms. In its most severe forms, ${ }^{1}$ Coronavirus Disease-19 (Covid-9) presents as an interstitial pneumonia that can develop an Acute Respiratory Distress Syndrome (ARDS), with severe hypoxemia refractory to oxygen administration, resulting in a high rate of early intubation and mechanical ventilation. $^{2}$

During the Covid-19 pandemic of february-april 2020, italian regional health systems and Emergency Medical Services (EMS)

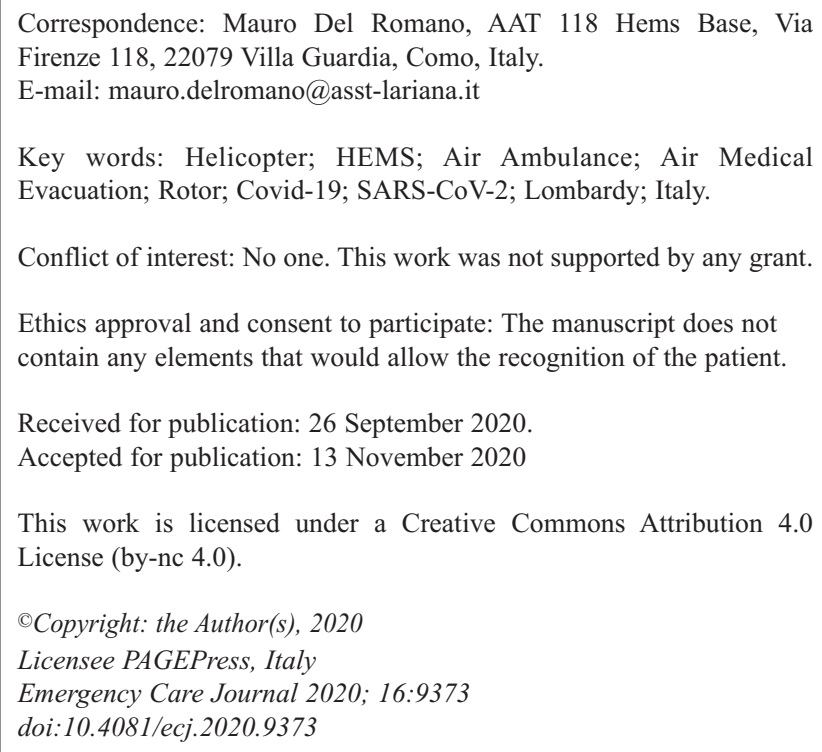

doi:10.4081/ecj.2020.9373

were under stress, and the long-range transport of both negative and positive COVID-19 patients became necessary to cope with the pressing demand for ICU beds.

The Como - Villa Guardia base is part of the AREU (Agency of the Lombardy region) Helicopter Emergency Medical Service (HEMS) that consists of a network of five helicopter bases located in Lombardy (Como, Sondrio, Bergamo, Brescia and Milan), two of which are also active at night with the NVG (Night Vision Goggles) system. The operations of our base are guaranteed by the helicopter HORUS 3, an AW139 (Leonardo) in HEMS configuration, certified for night flight and special NVG operations. The flight crew normally consists of two pilots, a Helicopter Hoist Operator (HHO), an intensive care physician, a critical area nurse and a mountain rescue technician.

In the absence of a relevant scientific literature on the aeromedical, non-military evacuation on rotary wing, ${ }^{3}$ and in the necessity to transfer Covid-19 patients from congested to less congested ICUs, we developed and applied specific procedures that take into consideration the pre-flight, in-flight and post-flight problems of this type of transport.

\section{Layout/Space Assessment}

The on-board equipment for the exclusive transport of Covid19 patients was completely revised, removing all the ordinary equipment and preparing a new checklist. The cockpit was sealed, thus isolating the medical compartment.

In order to maximize the protection of personnel and aircraft from the risk of contamination, the use of a high bio-containment cell was considered necessary. The high biocontainment cell IsoArk N36 (Beth-El Zikhron Yaaqov Ind. Ltd) is mounted directly on the aeronautical stretcher. The air is kept safe through an absolute (or a high-efficiency) filter. The patient is ventilated from taking-over to delivery with a Hamilton T1 ventilator, that can be used with high or low-pressure oxygen sources. The on-board oxygen supply is guaranteed by two fixed 5-liter cylinders and two removable 3-liter cylinders for the transfer from aircraft to hospital.

Monitoring is provided both with a Schiller Defigard Touch 7 monitor $\left(\mathrm{SpO}_{2}, \mathrm{ECG}, \mathrm{NIBP}\right.$ and $\left.\mathrm{EtCO}_{2}\right)$ placed outside the high bio-containment stretcher, and with a portable oximeter inside the stretcher. Infusions and drugs are regulated through 4 modular infusion systems (B. Braun Space) that can be used both inside and outside the stretcher. Nevertheless, an external infusion line is guaranteed for emergency therapy. The clinical backpack chosen for the transport is easily washable and small in size. The biocontainment cell also includes the equipment for possible emergency treatment (laryngeal tube and tension pneumothorax kit). 


\section{Coordination and planning}

The success of an HEMS mission in this scenario requires careful planning of all the different phases, including the organization, execution and termination of the operations; ${ }^{4}$ the assessment of transport feasibility should be made and shared between the operational centre of the Crisis Unit, the flight and medical crew, and the discharging and destination hospitals. In particular, prior to transport, patient's stability should be carefully assessed, as well as the possibility of intervening if clinical conditions should worsen during the transfer, ${ }^{4}$ given that after take-off accessing the patient in the biocontainment cell is difficult.

Although information from adequate randomized trials is still lacking, currently available data help outline the characteristics of intubated COVID-19 patients that can be transferred.

On the basis of experts' opinions, ${ }^{1,2}$ we drew up a series of recommendations as to whether or not a patient could be transported (Table 1), considering the complexity of transfer operations reported in the literature. 3,4

A meticulous optimization of the patient's condition before take-off is a key point to avoid injuries related to handling, turbulence and temperature changes during the flight ${ }^{5}$.

The discharging hospital is requested to have sedation ongoing before take-off, and to provide all the necessary equipment for his/her clinical care in order to minimize the time required to transfer the patient from the hospital to the helicopter (Table 2).

The goal during hand-over is not only to obtain all the information related to the patient's clinical situation, but also to prepare in advance all the monitoring devices, ventilator's circuit, infusion pumps and emergency medications.

\section{Missions performed}

During the period of March and April 2020, 11 patients were transported from Lombardy hospitals to several destinations across Italy, with a maximum distance of $286 \mathrm{NM}$ and mean distance of $156 \mathrm{NM}$; patient pick-up during daytime operations was performed both from standard and non-standard Helicopter Landing Sites (HLS) inserted in the route manual. As for NVG operations, only night standard HLS were used for both pick-up and release.

Despite the altitude reached in several missions (approx. $6000 / 7000 \mathrm{ft}$ ) to overcome the Apennine mountain range, there were no clinical respiratory problems related to the altitude. The ventilation strategy chosen was to keep the driving pressure at the lowest possible values, with an adequate inspired oxygen fraction to keep the oxygen saturation as close as possible to the pre-flight values throughout the transport.

The HEMS team always followed the patient from the moment of biocontainment until delivery to the destination ward, wearing adequate Personal Protection Equipment (PPE - Tyvek suit, FFP3 mask, gloves, face mask or glasses) ${ }^{6}$

Missions ended after arrival at the base, removal of PPE in a dedicated environment, helicopter sanitation through a procedure agreed with the operator and debriefing with the flight staff.

Helicopter decontamination was performed using a portable ozone diffusion system, ${ }^{7}$ positioned inside the cabin for 30 minutes behind closed doors with isoark stretcher and all the material used.

All the materials, including stretcher and electro-medical devices were subsequently washed out with a 1:9 solution of sodium hypochlorite and water.

\section{Table 1. Minimum requisites for the transport assessment of COVID-19-positive patients.}

General condition

Weight not exceeding $90 \mathrm{kgs}$, maximum height $\leq 180 \mathrm{~cm}$.

Hemodynamic stability.

Controlled temperature.

Ventilation

Orotracheal intubation or packed tracheostomy $>24 \mathrm{~h}$.

$\mathrm{PaO}_{2} / \mathrm{FiO}_{2}$ ratio $100 \mathrm{mmHg}$.

Ventilation: $\mathrm{FiO}_{2} \leq 0.70$, PEEP $\leq 12 \mathrm{cmH} 2 \mathrm{O}$, compensated EGA, $\mathrm{pH}>7.3, \mathrm{PaCO}_{2}<50 \mathrm{mmHg}$, lactates $\leq 1.5$.

Not subjected to pronation in the last 2 days*.

Current chest X-ray, no evidence of documented pneumothorax.

Recent chest CT scan with no evidence of massive pulmonary embolism.

Patient is responsive to recruitment maneuvers.

*It was decided to exclude patients recently subjected to prone positioning because they were considered at higher risk of worsening during transportation.

\section{Table 2. Patient preparation.}

Sedation preferably with benzodiazepine and morphine or propofol in continuous perfusion.

Adequate curarization for controlled ventilation.

Large caliber endotracheal tube.

Closed circuit bronchial aspiration probe.

Antibacterial / antiviral filter on respiratory circuit.

Central or peripheral venous access with extensions.

Nasal-gastric tube with possibility of aspiration.

Bladder catheter with empty collection bag.

Sealed clinical documentation.

Filled out MEDEVAC assessment form. 


\section{Conclusions}

The transport of patients affected by Covid-19 is a very special HEMS activity, and it is strictly dependent on an excellent synergy between all the actors involved, the experience of a highly qualified staff and of the emergency system network. ${ }^{8}$ The lack of specific civil literature on the topic led the Authors to develop a protocol in the attempt to standardize transport procedures for Covid19 patients with severe respiratory symptoms. During the first phase of response to the current pandemic, these procedures have allowed the safe transport of eleven patients with severe respiratory insufficiency from Covid-19, without significant complications for patients or health personnel. Further studies will be required to confirm the efficacy and safety of the procedures adopted and of the parameters used to validate patient transportability.

\section{References}

1. World Health Organization (WHO). Clinical management of COVID-19. WHO 2020. Available from: https://www.who.int/ publications-detail/clinical-management-of-severe-acute-respiratory-infection-when-novel-coronavirus-(ncov)-infectionis-suspected.
2. Meng L, Qiu H, Wan L, et al. Intubation and ventilation amid the COVID-19 Outbreak: Wuhan's Experience. Anesthesiol 2020;132:1317-32. doi:10.1097/ALN.0000000000003296

3. Gibbs SG, Herstein JJ, Le AB, et al. Review of literature for air medical evacuation high-level containment transport. Air Med J 2019;38:359-65. doi:10.1016/j.amj.2019.06.006

4. Nicol ED, Mepham S, Naylor J, et al. Aeromedical transfer of patients with viral hemorrhagic fever. Emerg Infect Dis 2019;25:5-14. doi:10.3201/eid2501.180662

5. Biselli R, Lastilla M, Arganese F, et al. The added value of preparedness for aeromedical evacuation of a patient with Ebola. Eur J Intern Med 2015;26:449-50. doi:10.1016/j.ejim. 2015.03.010

6. World Health Organization (WHO). Personal protective equipment in the context of filovirus disease outbreak response rapid advice guideline: summary of the recommendations. WHO 2014. Available from: https://apps.who.int/iris/handle/ $10665 / 137410$

7. Hudson JB, Manju S, Selvarani V. Development of a practical method for using ozone gas as a virus decontaminating agent. Sci Eng 2009;31:216-223. doi:10.1080/01919510902747969

8. Garibaldi B, Conger S, et al. 2019. Aeromedical evacuation of patients with contagious Infection. Aeromedical Evacuation 2019:317-35. doi:10.1007/978-3-030-15903-0_20 